\title{
Oscillation criteria for difference equations with non-monotone arguments
}

\author{
George E Chatzarakis ${ }^{1 *}$ and Leonid Shaikhet ${ }^{2}$
}

"Correspondence:
geaxatz@otenet.gr
${ }^{1}$ Department of Electrical and
Electronic Engineering Educators,
School of Pedagogical and
Technological Education (ASPETE),
N. Heraklio, Athens, 14121, Greece
Full list of author information is
available at the end of the article

"Correspondence: geaxatz@otenet.g

Department of Electrical School of Pedagogical and Technological Education (ASPETE), Full list of author information is ave end the article

\begin{abstract}
This paper is concerned with the oscillatory behavior of first-order retarded [advanced] difference equation of the form

$$
\Delta x(n)+p(n) x(\tau(n))=0, \quad n \in \mathbb{N}_{0} \quad[\nabla x(n)-q(n) x(\sigma(n))=0, n \in \mathbb{N}],
$$

where $(p(n))_{n \geq 0}\left[(q(n))_{n \geq 1}\right]$ is a sequence of nonnegative real numbers and $\tau(n)[\sigma(n)]$ is a non-monotone sequence of integers such that $\tau(n) \leq n-1$, for $n \in \mathbb{N}_{0}$ and $\lim _{n \rightarrow \infty} \tau(n)=\infty[\sigma(n) \geq n+1$, for $n \in \mathbb{N}]$. Sufficient conditions, involving lim sup, which guarantee the oscillation of all solutions are established. These conditions improve all previous well-known results in the literature. Also, using algorithms on MATLAB software, examples illustrating the significance of the results are given.
\end{abstract}

MSC: 39A10; 39A21

Keywords: difference equations; non-monotone arguments; retarded arguments; advanced arguments; oscillation; Grönwall inequality

\section{Introduction}

The paper deals with the difference equation with a single variable retarded argument of the form

$$
\Delta x(n)+p(n) x(\tau(n))=0, \quad n \in \mathbb{N}_{0},
$$

and the (dual) difference equation with a single variable advanced argument of the form

$$
\nabla x(n)-q(n) x(\sigma(n))=0, \quad n \in \mathbb{N}
$$

where $\mathbb{N}_{0}$ and $\mathbb{N}$ are the sets of nonnegative integers and positive integers, respectively.

Equations $(E)$ and $\left(E^{\prime}\right)$ are studied under the following assumptions: everywhere $(p(n))_{n \geq 0}$ and $(q(n))_{n \geq 1}$ are sequences of nonnegative real numbers, $(\tau(n))_{n \geq 0}$ is a sequence of integers such that

$$
\tau(n) \leq n-1, \quad \forall n \in \mathbb{N}_{0} \quad \text { and } \quad \lim _{n \rightarrow \infty} \tau(n)=\infty,
$$

(c) The Author(s) 2017. This article is distributed under the terms of the Creative Commons Attribution 4.0 International License (http://creativecommons.org/licenses/by/4.0/), which permits unrestricted use, distribution, and reproduction in any medium, provided you give appropriate credit to the original author(s) and the source, provide a link to the Creative Commons license, and indicate if changes were made. 
and $(\sigma(n))_{n \geq 1}$ is a sequence of integers such that

$$
\sigma(n) \geq n+1, \quad \forall n \in \mathbb{N} .
$$

Here, $\Delta$ denotes the forward difference operator $\Delta x(n)=x(n+1)-x(n)$ and $\nabla$ corresponds to the backward difference operator $\nabla x(n)=x(n)-x(n-1)$.

Set

$$
w=-\min _{n \geq 0} \tau(n) .
$$

Clearly, $w$ is a finite positive integer if (1.1) holds.

By a solution of $(E)$, we mean a sequence of real numbers $(x(n))_{n \geq-w}$ which satisfies (E) for all $n \geq 0$. It is clear that, for each choice of real numbers $c_{-w}, c_{-w+1}, \ldots, c_{-1}, c_{0}$, there exists a unique solution $(x(n))_{n \geq-w}$ of $(\mathrm{E})$ which satisfies the initial conditions $x(-w)=c_{-w}, x(-w+$ 1) $=c_{-w+1}, \ldots, x(-1)=c_{-1}, x(0)=c_{0}$.

By a solution of $\left(E^{\prime}\right)$, we mean a sequence of real numbers $(x(n))_{n \geq 0}$ which satisfies $\left(E^{\prime}\right)$ for all $n \geq 1$.

A solution $(x(n))_{n \geq-w}\left(\right.$ or $\left.(x(n))_{n \geq 0}\right)$ of $(\mathrm{E})$ (or $\left.\left(\mathrm{E}^{\prime}\right)\right)$ is called oscillatory, if the terms $x(n)$ of the sequence are neither eventually positive nor eventually negative. Otherwise, the solution is said to be nonoscillatory. An equation is oscillatory if all its solutions oscillate.

In the last few decades, the oscillatory behavior and the existence of positive solutions of difference equations with deviating arguments have been extensively studied; see, for example, papers [1-19] and the references cited therein. Most of these papers concern the special case where the arguments are nondecreasing, while a small number of these papers are dealing with the general case where the arguments are non-monotone. See, for example, $[1-3,11,15]$ and the references cited therein. By the consideration of nonmonotone arguments of other than the pure mathematical interest, it approximates the natural phenomena described by equation of the type (E) or $\left(E^{\prime}\right)$. That is because there are always natural disturbances (e.g. noise in communication systems) that affect all the parameters of the equation and therefore the fair (from a mathematical point of view) monotone arguments become non-monotone almost always.

\subsection{Retarded difference equations}

In 2008 Chatzarakis, Koplatadze and Stavroulakis $[4,5]$ proved that, if

$$
\limsup _{n \rightarrow \infty} \sum_{j=h(n)}^{n} p(j)>1,
$$

where $h(n)=\max _{0 \leq s \leq n} \tau(s)$, or

$$
\liminf _{n \rightarrow \infty} \sum_{j=\tau(n)}^{n-1} p(j)>\frac{1}{e}
$$

then all solutions of (E) oscillate. 
It is obvious that there is a gap between the conditions (1.2) and (1.3) when the limit

$$
\lim _{n \rightarrow \infty} \sum_{j=\tau(n)}^{n-1} p(j)
$$

does not exist. How to fill this gap is an interesting problem which has been investigated by several authors. For example, in 2009, Chatzarakis, Philos and Stavroulakis [6] proved that if

$$
\limsup _{n \rightarrow \infty} \sum_{j=h(n)}^{n} p(j)>1-\frac{1-a-\sqrt{1-2 a-a^{2}}}{2},
$$

where $a=\liminf _{n \rightarrow \infty} \sum_{j=\tau(n)}^{n-1} p(j)$, then all solutions of (E) oscillate.

In 2011, Braverman and Karpuz [3] proved that if

$$
\limsup _{n \rightarrow \infty} \sum_{j=h(n)}^{n} p(j) \prod_{i=\tau(j)}^{h(n)-1} \frac{1}{1-p(i)}>1,
$$

then all solutions of (E) oscillate, while, in 2014, Stavroulakis [15], improved (1.5) to

$$
\limsup _{n \rightarrow \infty} \sum_{j=h(n)}^{n} p(j) \prod_{i=\tau(j)}^{h(n)-1} \frac{1}{1-p(i)}>1-\frac{1-a-\sqrt{1-2 a-a^{2}}}{2} .
$$

In 2015, Braverman, Chatzarakis, and Stavroulakis [2] proved that if for some $r \in \mathbb{N}$

$$
\limsup _{n \rightarrow \infty} \sum_{j=h(n)}^{n} p(j) a_{r}^{-1}(h(n), \tau(j))>1,
$$

or

$$
\limsup _{n \rightarrow \infty} \sum_{j=h(n)}^{n} p(j) a_{r}^{-1}(h(n), \tau(j))>1-\frac{1-a-\sqrt{1-2 a-a^{2}}}{2},
$$

where

$$
a_{1}(n, k)=\prod_{i=k}^{n-1}[1-p(i)], \quad a_{r+1}(n, k)=\prod_{i=k}^{n-1}\left[1-p(i) a_{r}^{-1}(i, \tau(i))\right],
$$

then all solutions of (E) oscillate.

Recently, Asteris and Chatzarakis [1] proved that if for some $\ell \in \mathbb{N}$

$$
\limsup _{n \rightarrow \infty} \sum_{i=h(n)}^{n} p(i) \prod_{j=\tau(i)}^{h(n)-1} \frac{1}{1-p_{\ell}(j)}>1,
$$

where

$$
p_{\ell}(n)=p(n)\left[1+\sum_{i=\tau(n)}^{n-1} p(i) \prod_{j=\tau(i)}^{h(n)-1} \frac{1}{1-p_{\ell-1}(j)}\right]
$$

with $p_{0}(n)=p(n)$, then all solutions of $(\mathrm{E})$ oscillate. 


\subsection{Advanced difference equations}

In 2012, Chatzarakis and Stavroulakis [7] proved that, if

$$
\limsup _{n \rightarrow \infty} \sum_{i=n}^{\rho(n)} q(i)>1
$$

or

$$
\limsup _{n \rightarrow \infty} \sum_{i=n}^{\rho(n)} q(i)>1-(1-\sqrt{1-b})^{2}
$$

where $\rho(n)=\min _{s \geq n} \sigma(s)$ and $b=\liminf _{n \rightarrow \infty} \sum_{i=n+1}^{\sigma(n)} p(i)$, then all solutions of $\left(\mathrm{E}^{\prime}\right)$ oscillate. In 2015, Braverman, Chatzarakis, and Stavroulakis [2] proved that if for some $r \in \mathbb{N}$

$$
\limsup _{n \rightarrow \infty} \sum_{j=n}^{\rho(n)} q(j) b_{r}^{-1}(\rho(n), \sigma(j))>1
$$

or

$$
\limsup _{n \rightarrow \infty} \sum_{j=n}^{\rho(n)} q(j) b_{r}^{-1}(\rho(n), \sigma(j))>1-\frac{1-b-\sqrt{1-2 b-b^{2}}}{2},
$$

where

$$
\begin{aligned}
& b_{1}(n, k)=\prod_{i=n+1}^{k}[1-q(i)], \\
& b_{r+1}(n, k)=\prod_{i=n+1}^{k}\left[1-q(i) b_{r}^{-1}(i, \sigma(i))\right]
\end{aligned}
$$

and $b=\liminf _{n \rightarrow \infty} \sum_{i=n+1}^{\sigma(n)} p(i)$, then all solutions of $\left(\mathrm{E}^{\prime}\right)$ oscillate.

Recently, Asteris and Chatzarakis [1] proved that if for some $\ell \in \mathbb{N}$

$$
\limsup _{n \rightarrow \infty} \sum_{i=n}^{\rho(n)} q(i) \prod_{j=\rho(n)+1}^{\sigma(i)} \frac{1}{1-q_{\ell}(j)}>1,
$$

where

$$
q_{\ell}(n)=q(n)\left[1+\sum_{i=n+1}^{\rho(n)} q(i) \prod_{j=\rho(n)+1}^{\sigma(i)} \frac{1}{1-q_{\ell-1}(j)}\right]
$$

with $q_{0}(n)=q(n)$, then all solutions of $\left(\mathrm{E}^{\prime}\right)$ oscillate.

In this paper we study further $(E)$ and $\left(E^{\prime}\right)$ and derive new sufficient oscillation conditions. Examples illustrate cases when the results of the present paper imply oscillation while previously known results fail. 


\section{Main results}

\subsection{Retarded difference equations}

We study further (E) and derive a new sufficient oscillation condition, involving lim sup, which essentially improves all the previous results.

Let

$$
h(n)=\max _{0 \leq s \leq n} \tau(s), \quad n \geq 0
$$

Clearly, the sequence $h(n)$ is nondecreasing and $\tau(n) \leq h(n) \leq n-1$ for all $n \geq 0$.

The proof of our main result is essentially based on the following lemmas.

Lemma 1 Assume that (1.1) holds and

$$
0<a:=\liminf _{n \rightarrow \infty} \sum_{j=\tau(n)}^{n-1} p(j) .
$$

Then we have

$$
\liminf _{n \rightarrow \infty} \sum_{j=h(n)}^{n-1} p(j)=\liminf _{n \rightarrow \infty} \sum_{j=\tau(n)}^{n-1} p(j)=a,
$$

where $h(n)$ is defined by (2.1).

Proof Since $h(n)$ is nondecreasing and $\tau(n) \leq h(n) \leq n-1$ for all $n \geq 0$, we have

$$
\sum_{j=h(n)}^{n-1} p(j) \leq \sum_{j=\tau(n)}^{n-1} p(j)
$$

Therefore

$$
\liminf _{n \rightarrow \infty} \sum_{j=h(n)}^{n-1} p(j) \leq \liminf _{n \rightarrow \infty} \sum_{j=\tau(n)}^{n-1} p(j) .
$$

If (2.2) does not hold, then there exist $a^{\prime}>0$ and a subsequence $(\theta(n))$ such that $\theta(n) \rightarrow \infty$ as $n \rightarrow \infty$ and

$$
\lim _{n \rightarrow \infty} \sum_{j=h(\theta(n))}^{\theta(n)-1} p(j) \leq a^{\prime}<a .
$$

But $h(\theta(n))=\max _{0 \leq s \leq \theta(n)} \tau(s)$, hence there exists $\theta^{\prime}(n) \leq \theta(n), \theta^{\prime}(n) \in \mathbb{N}_{0}$ such that $h(\theta(n))=\tau\left(\theta^{\prime}(n)\right)$, and consequently

$$
\sum_{j=h(\theta(n))}^{\theta(n)-1} p(j)=\sum_{j=\tau\left(\theta^{\prime}(n)\right)}^{\theta(n)-1} p(j) \geq \sum_{j=\tau\left(\theta^{\prime}(n)\right)}^{\theta^{\prime}(n)-1} p(j) .
$$


It follows that $\left(\sum_{j=\tau\left(\theta^{\prime}(n)\right)}^{\theta^{\prime}(n)-1} p(j)\right)_{n=1}^{\infty}$ is a bounded sequence having a convergent subsequence, say

$$
\sum_{j=\tau\left(\theta^{\prime}\left(n_{k}\right)\right)}^{\theta^{\prime}\left(n_{k}\right)-1} p(j) \rightarrow c \leq a^{\prime}, \quad \text { as } k \rightarrow \infty
$$

which implies that

$$
\liminf _{n \rightarrow \infty} \sum_{j=\tau(n)}^{n-1} p(j) \leq a^{\prime}<a
$$

This contradicts (2.2).

The proof of the lemma is complete.

Lemma 2 ([6], Lemma 2.1) In addition to hypothesis (1.1), assume that $h(n)$ is defined by $(2.1)$

$$
0<a:=\liminf _{n \rightarrow \infty} \sum_{j=\tau(n)}^{n-1} p(j) \leq \frac{1}{e}
$$

and $x(n)$ is an eventually positive solution of $(\mathrm{E})$. Then

$$
\liminf _{n \rightarrow \infty} \frac{x(n+1)}{x(h(n))} \geq \frac{1-a-\sqrt{1-2 a-a^{2}}}{2}
$$

Theorem 1 Assume that (1.1) holds, $h(n)$ is defined by (2.1) and a by (2.3). Iffor some $\ell \in \mathbb{N}$

$$
\limsup _{n \rightarrow \infty} \sum_{i=h(n)}^{n} p(i) \prod_{j=\tau(i)}^{h(n)-1} \frac{1}{1-p_{\ell}(j)}>1-\frac{1-a-\sqrt{1-2 a-a^{2}}}{2}
$$

where $p_{\ell}(n)$ is defined by (1.11), then all solutions of (E) oscillate.

Proof Assume, for the sake of contradiction, that $(x(n))_{n \geq-w}$ is a nonoscillatory solution of $(\mathrm{E})$. Then it is either eventually positive or eventually negative. As $(-x(n))_{n \geq-w}$ is also a solution of $(\mathrm{E})$, we may restrict ourselves only to the case where $x(n)>0$ for all large $n$. Let $n_{1} \geq-w$ be an integer such that $x(n)>0$ for all $n \geq n_{1}$. Then, there exists $n_{2} \geq n_{1}$ such that $x(\tau(n))>0, \forall n \geq n_{2}$. In view of this, equation (E) becomes

$$
\Delta x(n)=-p(n) x(\tau(n)) \leq 0, \quad \forall n \geq n_{2}
$$

which means that the sequence $(x(n))$ is eventually decreasing.

Therefore, since $\tau(n)<n$, (E) implies

$$
\Delta x(n)+p(n) x(n) \leq 0 .
$$


Applying the discrete Grönwall inequality, we obtain

$$
x(k) \geq x(n) \prod_{i=k}^{n-1} \frac{1}{1-p(i)}, \quad \text { for all } n \geq k \geq n_{2} .
$$

Summing up (E) from $\tau(n)$ to $n-1$, we have

$$
x(n)-x(\tau(n))+\sum_{i=\tau(n)}^{n-1} p(i) x(\tau(i))=0
$$

Since $\tau(i) \leq h(i) \leq h(n),(2.7)$ and (2.8) give

$$
x(n)-x(\tau(n))+x(h(n)) \sum_{i=\tau(n)}^{n-1} p(i) \prod_{j=\tau(i)}^{h(n)-1} \frac{1}{1-p(j)} \leq 0 .
$$

Multiplying the last inequality by $p(n)$, we get

$$
p(n) x(n)-p(n) x(\tau(n))+p(n) x(h(n)) \sum_{i=\tau(n)}^{n-1} p(i) \prod_{j=\tau(i)}^{h(n)-1} \frac{1}{1-p(j)} \leq 0
$$

which, in view of (E), becomes

$$
\Delta x(n)+p(n) x(n)+p(n) x(h(n)) \sum_{i=\tau(n)}^{n-1} p(i) \prod_{j=\tau(i)}^{h(n)-1} \frac{1}{1-p(j)} \leq 0 .
$$

Since $h(n)<n$, the last inequality gives

$$
\Delta x(n)+p(n) x(n)+p(n) x(n) \sum_{i=\tau(n)}^{n-1} p(i) \prod_{j=\tau(i)}^{h(n)-1} \frac{1}{1-p(j)} \leq 0,
$$

or

$$
\Delta x(n)+p(n)\left[1+\sum_{i=\tau(n)}^{n-1} p(i) \prod_{j=\tau(i)}^{h(n)-1} \frac{1}{1-p(j)}\right] x(n) \leq 0 .
$$

Therefore

$$
\Delta x(n)+p_{1}(n) x(n) \leq 0,
$$

where

$$
p_{1}(n)=p(n)\left[1+\sum_{i=\tau(n)}^{n-1} p(i) \prod_{j=\tau(i)}^{h(n)-1} \frac{1}{1-p(j)}\right]
$$


Repeating the above argument leads to a new estimate,

$$
\Delta x(n)+p_{2}(n) x(n) \leq 0
$$

where

$$
p_{2}(n)=p(n)\left[1+\sum_{i=\tau(n)}^{n-1} p(i) \prod_{j=\tau(i)}^{h(n)-1} \frac{1}{1-p_{1}(j)}\right]
$$

Continuing by induction, for sufficiently large $n$ we get

$$
\Delta x(n)+p_{\ell}(n) x(n) \leq 0
$$

where

$$
p_{\ell}(n)=p(n)\left[1+\sum_{i=\tau(n)}^{n-1} p(i) \prod_{j=\tau(i)}^{h(n)-1} \frac{1}{1-p_{\ell-1}(j)}\right]
$$

Clearly, by the Grönwall inequality, we have

$$
x(\tau(i)) \geq x(h(n)) \prod_{j=\tau(i)}^{h(n)-1} \frac{1}{1-p_{\ell}(j)}
$$

Summing up (E) from $h(n)$ to $n$, we have

$$
x(n+1)-x(h(n))+\sum_{i=h(n)}^{n} p(i) x(\tau(i))=0 .
$$

Combining (2.9) and (2.10), we have

$$
x(n+1)-x(h(n))+x(h(n)) \sum_{i=h(n)}^{n} p(i) \prod_{j=\tau(i)}^{h(n)-1} \frac{1}{1-p_{\ell}(j)} \leq 0
$$

or

$$
\sum_{i=h(n)}^{n} p(i) \prod_{j=\tau(i)}^{h(n)-1} \frac{1}{1-p_{\ell}(j)} \leq 1-\frac{x(n+1)}{x(h(n))}
$$

Therefore

$$
\limsup _{n \rightarrow \infty} \sum_{i=h(n)}^{n} p(i) \prod_{j=\tau(i)}^{h(n)-1} \frac{1}{1-p_{\ell}(j)} \leq 1-\liminf _{n \rightarrow \infty} \frac{x(n+1)}{x(h(n))} .
$$


By combining Lemmas 1 and 2, it becomes obvious that inequality (2.4) is fulfilled. So, (2.11) leads to

$$
\limsup _{n \rightarrow \infty} \sum_{i=h(n)}^{n} p(i) \prod_{j=\tau(i)}^{h(n)-1} \frac{1}{1-p_{\ell}(j)} \leq 1-\frac{1-a-\sqrt{1-2 a-a^{2}}}{2}
$$

which contradicts (2.5). The proof of the theorem is complete.

Example 1 Consider the retarded difference equation

$$
\Delta x(n)+\frac{179}{1,000} x(\tau(n))=0, \quad n \in \mathbb{N}_{0},
$$

with (see Figure 1(a))

$$
\tau(n)= \begin{cases}n-3, & \text { if } n=5 \mu \\ n-1, & \text { if } n=5 \mu+1 \\ n-1, & \text { if } n=5 \mu+2, \\ n-4, & \text { if } n=5 \mu+3 \\ n-3, & \text { if } n=5 \mu+4\end{cases}
$$

By (2.1), we see (Figure 1(b)) that

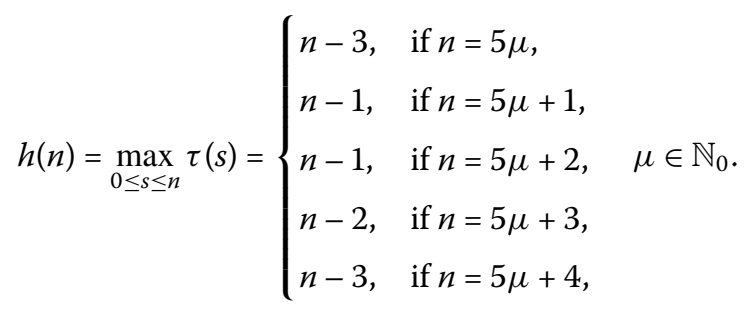

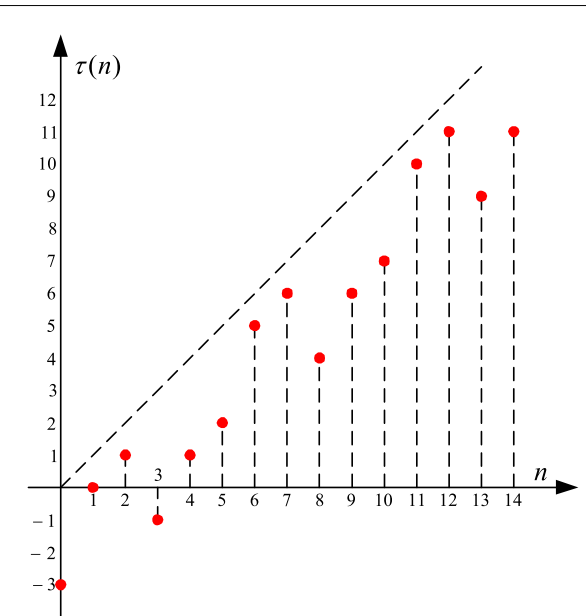

(a)

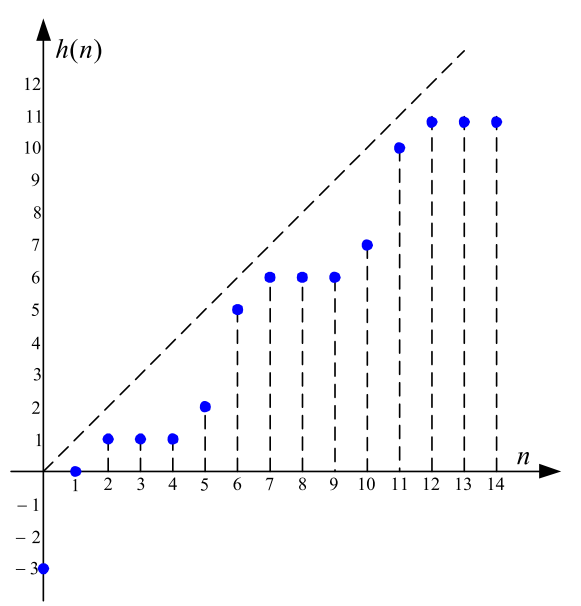

(b)

Figure 1 The graphs of $\tau(n)$ and $h(n)$. 
Observe that the function $F: \mathbb{N}_{0} \rightarrow \mathbb{R}_{+}$defined as

$$
F_{\ell}(n)=\sum_{i=h(n)}^{n} p(i) \prod_{j=\tau(i)}^{h(n)-1} \frac{1}{1-p_{\ell}(j)}
$$

attains its maximum at $n=5 \mu+4, \mu \in \mathbb{N}_{0}$, for every $\ell \in \mathbb{N}$. Specifically, by using an algorithm of Matlab software, we obtain

$$
\begin{aligned}
F_{1}(n) & =\sum_{i=h(n)}^{n} p(i) \prod_{j=\tau(i)}^{h(n)-1} \frac{1}{1-p_{1}(j)} \\
& =\sum_{i=h(n)}^{n} p(i) \prod_{j=\tau(i)}^{h(n)-1} \frac{1}{1-p(j)\left[1+\sum_{k=\tau(j)}^{j-1} p(k) \prod_{m=\tau(k)}^{h(j)-1} \frac{1}{1-p(m)}\right]} \\
& =\sum_{i=5 \mu+1}^{5 \mu+4} \frac{179}{1,000} \prod_{j=\tau(i)}^{3 \mu-2} \frac{1}{1-\frac{179}{1,000}\left[1+\sum_{k=\tau(j)}^{j-1} \frac{179}{1,000} \prod_{m=\tau(k)}^{h(j)-1} \frac{1}{1-\frac{179}{1,000}}\right]} \\
& \simeq 0.9913 .
\end{aligned}
$$

Thus

$$
\limsup _{n \rightarrow \infty} F_{1}(n)=\limsup _{n \rightarrow \infty} \sum_{i=h(n)}^{n} p(i) \prod_{j=\tau(i)}^{h(n)-1} \frac{1}{1-p_{1}(j)} \simeq 0.9913
$$

Since

$$
a=\liminf _{n \rightarrow \infty} \sum_{j=\tau(n)}^{n-1} p(j)=\liminf _{n \rightarrow \infty} \sum_{j=5 \mu}^{5 \mu} p(j)=0.179
$$

we have

$$
0.9913>1-\frac{1-a-\sqrt{1-2 a-a^{2}}}{2} \simeq 0.9799
$$

that is, condition (2.5) of Theorem 1 is satisfied for $\ell=1$. Therefore, all solutions of (2.12) oscillate.

Observe, however, that

$$
\begin{aligned}
& \limsup _{n \rightarrow \infty} \sum_{j=h(n)}^{n} p(j)=\limsup _{\mu \rightarrow \infty} \sum_{i=5 \mu+1}^{5 \mu+4} p(j)=4 \cdot 0.179=0.716<1, \\
& a=0.179<\frac{1}{e} \\
& 0.716<1-\frac{1-a-\sqrt{1-2 a-a^{2}}}{2} \simeq 0.9799
\end{aligned}
$$




$$
\begin{aligned}
& \limsup _{n \rightarrow \infty} \sum_{j=h(n)}^{n} p(j) \prod_{i=\tau(j)}^{h(n)-1} \frac{1}{1-p(i)} \\
& =\limsup _{\mu \rightarrow \infty} \sum_{j=5 \mu+1}^{5 \mu+4} 0.179 \prod_{i=\tau(j)}^{5 \mu} \frac{1}{1-0.179} \\
& =0.179 \cdot \limsup _{\mu \rightarrow \infty}\left\{\prod_{i=\tau(5 \mu+1)}^{5 \mu} \frac{1}{1-0.179}+\prod_{i=\tau(5 \mu+2)}^{5 \mu} \frac{1}{1-0.179}\right. \\
& \left.+\prod_{i=\tau(5 \mu+3)}^{5 \mu} \frac{1}{1-0.179}+\prod_{i=\tau(5 \mu+4)}^{5 \mu} \frac{1}{1-0.179}\right\} \\
& =0.179 \cdot \limsup _{\mu \rightarrow \infty}\left\{\prod_{i=5 \mu}^{5 \mu} \frac{1}{1-0.179}+\prod_{i=5 \mu+1}^{5 \mu} \frac{1}{1-0.179}+\prod_{i=5 \mu-1}^{5 \mu} \frac{1}{1-0.179}\right. \\
& \left.+\prod_{i=5 \mu+1}^{5 \mu} \frac{1}{1-0.179}\right\} \\
& =0.179 \cdot\left\{\frac{1}{1-0.179}+1+\left(\frac{1}{1-0.179}\right)^{2}+1\right\} \simeq 0.8416<1 \text {, } \\
& 0.8416<1-\frac{1-a-\sqrt{1-2 a-a^{2}}}{2} \simeq 0.9799 \text {, }
\end{aligned}
$$

That is, conditions (1.2), (1.3), (1.4), (1.5) $\equiv(1.7)$ (for $r=1),(1.6) \equiv(1.8)$ (for $r=1)$ and (1.10) are not satisfied.

Figure 2, illustrates that the three different solutions of (2.12), as predicted, appear to be oscillatory.

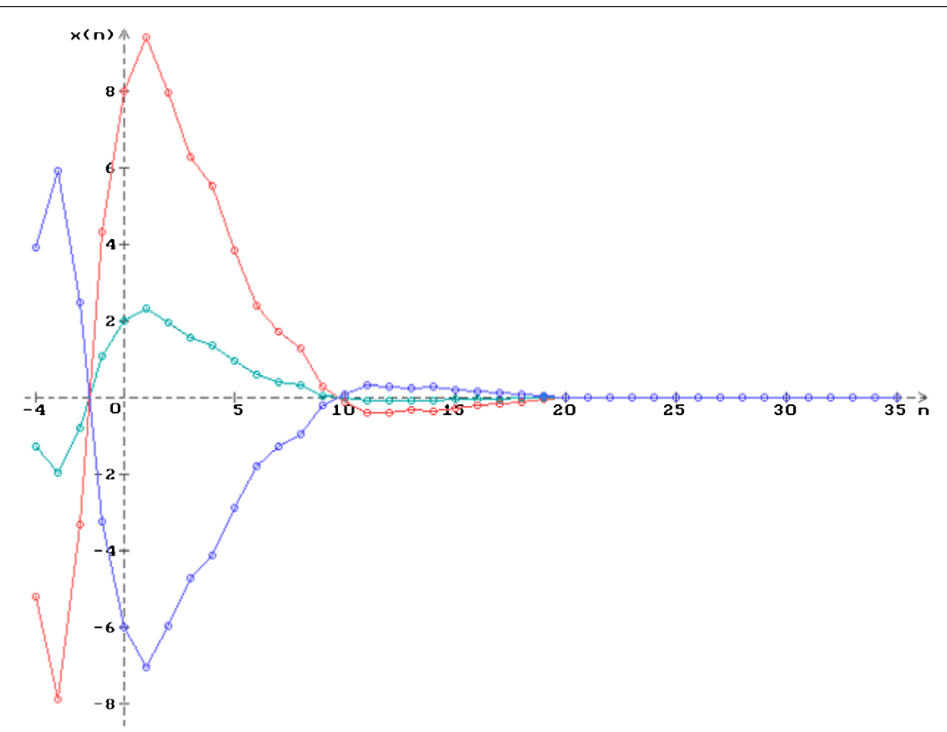

Figure 2 Three solutions of (2.12). 
Notation It is worth noting that the improvement of condition (2.5) to the corresponding condition (1.4) is significant, approximately $38.45 \%$, if we compare the values on the lefthand side of these conditions. Also, the improvement compared to condition (1.5) (or (1.6) or (1.7) or (1.8)) is very satisfactory, around $17.8 \%$. Also, observe that conditions (1.7), (1.8), and (1.10) do not lead to oscillation for the first iteration. On the contrary, condition (2.5) is satisfied from the first iteration. This means that our condition is better and much faster than (1.7), (1.8), and (1.10).

\subsection{Advanced difference equations}

A similar oscillation theorem for the (dual) advanced difference equation $\left(E^{\prime}\right)$ can be derived easily. The proof of this theorem is omitted, since it is quite similar to the proof for a retarded equation.

Let

$$
\rho(n)=\min _{s \geq n} \sigma(s), \quad n \geq 1 .
$$

Clearly, the sequence $\rho(n)$ is nondecreasing and $\sigma(n) \geq \rho(n) \geq n+1$ for all $n \geq 1$.

Theorem 2 Assume that $\left(1.1^{\prime}\right)$ holds and $\rho(n)$ is defined by (2.13). If for some $\ell \in \mathbb{N}$

$$
\limsup _{n \rightarrow \infty} \sum_{i=n}^{\rho(n)} q(i) \prod_{j=\rho(n)+1}^{\sigma(i)} \frac{1}{1-q_{\ell}(j)}>1-\frac{1-b-\sqrt{1-2 b-b^{2}}}{2}
$$

where $q_{\ell}(n)$ is defined by (1.18) and $0<b=\liminf _{n \rightarrow \infty} \sum_{i=n+1}^{\sigma(n)} p(i) \leq 1 / e$, then all solutions of $\left(\mathrm{E}^{\prime}\right)$ oscillate.

Example 2 Consider the advanced difference equation

$$
\nabla x(n)-\frac{341}{2,000} x(\sigma(n))=0, \quad n \in \mathbb{N}_{0},
$$

with (see Figure 3(a))

$$
\sigma(n)= \begin{cases}n+1, & \text { if } n=5 \mu \\ n+5, & \text { if } n=5 \mu+1, \\ n+1, & \text { if } n=5 \mu+2, \quad \mu \in \mathbb{N}_{0} . \\ n+1, & \text { if } n=5 \mu+3, \\ n+2, & \text { if } n=5 \mu+4,\end{cases}
$$

By (2.13), we see (Figure 3(b)) that

$$
\rho(n)=\min _{s \geq n} \sigma(s)= \begin{cases}n+1, & \text { if } n=5 \mu, \\ n+2, & \text { if } n=5 \mu+1, \\ n+1, & \text { if } n=5 \mu+2, \quad \mu \in \mathbb{N}_{0} . \\ n+1, & \text { if } n=5 \mu+3, \\ n+2, & \text { if } n=5 \mu+4,\end{cases}
$$




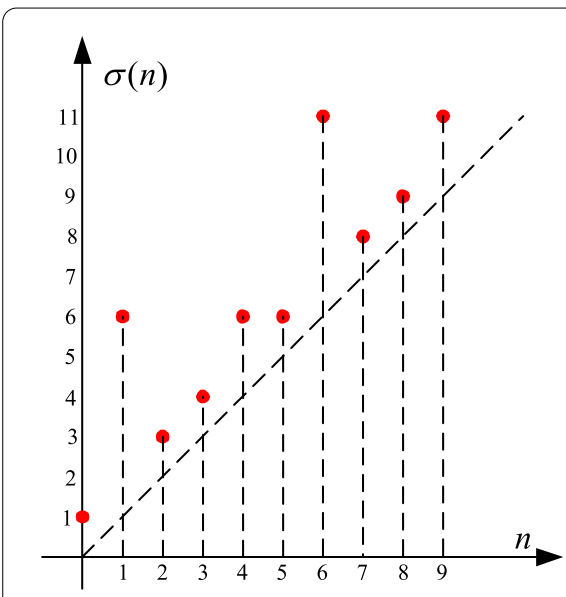

(a)

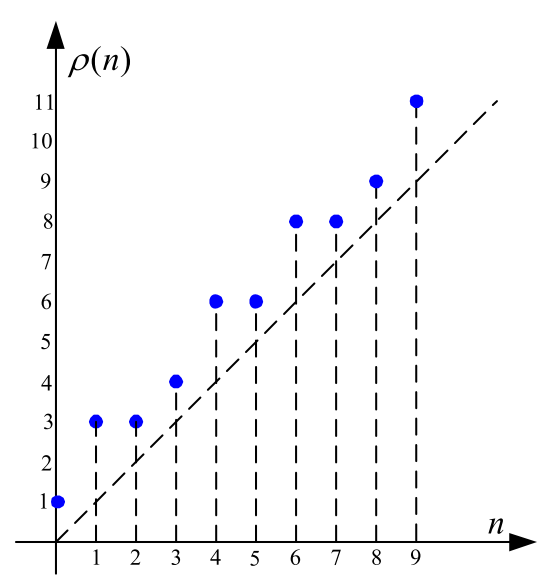

(b)

Figure 3 The graphs of $\sigma(n)$ and $\rho(n)$.

Observe that the function $F: \mathbb{N}_{0} \rightarrow \mathbb{R}_{+}$defined as

$$
F_{\ell}(n)=\sum_{i=n}^{\rho(n)} q(i) \prod_{j=\rho(n)+1}^{\sigma(i)} \frac{1}{1-q_{\ell}(j)}
$$

attains its maximum at $n=5 \mu+1, \mu \in \mathbb{N}_{0}$, for every $\ell \in \mathbb{N}$. Specifically, by using an algorithm of Matlab software, we obtain

$$
\begin{aligned}
F_{1}(n) & =\sum_{i=n}^{\rho(n)} q(i) \prod_{j=\rho(n)+1}^{\sigma(i)} \frac{1}{1-q_{1}(j)} \\
& =\sum_{i=n}^{\rho(n)} q(i) \prod_{j=\rho(n)+1}^{\sigma(i)} \frac{1}{1-q(j)\left[1+\sum_{k=j+1}^{\sigma(j)} q(k) \prod_{m=\rho(j)+1}^{\sigma(k)} \frac{1}{1-q(m)}\right]} \\
& =\sum_{i=5 \mu+1}^{5 \mu+3} \frac{341}{2,000} \prod_{j=5 \mu+4}^{\sigma(i)} \frac{1}{1-\frac{341}{2,000}\left[1+\sum_{k=j+1}^{\sigma(j)} \frac{341}{2,000} \prod_{m=\rho(j)+1}^{\sigma(k)} \frac{1}{1-\frac{341}{2,000}}\right]} \simeq 0.9872 .
\end{aligned}
$$

Thus

$$
\limsup _{n \rightarrow \infty} F_{1}(n)=\limsup _{n \rightarrow \infty} \sum_{i=n}^{\rho(n)} q(i) \prod_{j=\rho(n)+1}^{\sigma(i)} \frac{1}{1-q_{1}(j)} \simeq 0.9872 .
$$

Since

$$
b=\liminf _{n \rightarrow \infty} \sum_{i=n+1}^{\sigma(n)} q(i)=\liminf _{n \rightarrow \infty} \sum_{i=5 \mu+1}^{5 \mu+1} q(i)=\frac{341}{2,000}=0.1705,
$$

we have

$$
0.9872>1-\frac{1-b-\sqrt{1-2 b-b^{2}}}{2} \simeq 0.9821
$$


that is, condition (2.14) of Theorem 2 is satisfied for $\ell=1$. Therefore, all solutions of (2.15) oscillate.

Observe, however, that

$$
\begin{aligned}
& \limsup _{n \rightarrow \infty} \sum_{i=n}^{\rho(n)} q(i)=\limsup _{\mu \rightarrow \infty} \sum_{i=5 \mu+1}^{5 \mu+3} q(i)=3 \cdot \frac{341}{2,000}=0.5115<1, \\
& 0.5115<1-(1-\sqrt{1-b})^{2} \simeq 0.9920, \\
& \limsup _{n \rightarrow \infty} \sum_{j=n}^{\rho(n)} q(j) b_{1}^{-1}(\rho(n), \sigma(j)) \\
& =\limsup _{\mu \rightarrow \infty} \sum_{j=5 \mu+1}^{5 \mu+3} \frac{341}{2,000} \prod_{i=5 \mu+4}^{\sigma(j)} \frac{1}{1-\frac{341}{2,000}} \\
& \quad=\frac{341}{2,000} \cdot \limsup _{\mu \rightarrow \infty}\left\{\prod_{i=5 \mu+4}^{\sigma(5 \mu+1)} \frac{1}{1-\frac{341}{2,000}}+\prod_{i=5 \mu+4}^{\sigma(5 \mu+2)} \frac{1}{1-\frac{341}{2,000}}+\prod_{i=5 \mu+4}^{\sigma(5 \mu+3)} \frac{1}{1-\frac{341}{2,000}}\right\} \\
& \quad=\frac{341}{2,000} \cdot \limsup _{\mu \rightarrow \infty}\left\{\prod_{i=5 \mu+4}^{5 \mu+6} \frac{1}{1-\frac{341}{2,000}}+\prod_{i=5 \mu+4}^{5 \mu+3} \frac{1}{1-\frac{341}{2,000}}+\prod_{i=5 \mu+4}^{5 \mu+4} \frac{1}{1-\frac{341}{2,000}}\right\} \\
& \quad=\frac{341}{2,000} \cdot\left\{\frac{1}{\left(1-\frac{341}{2,000}\right)^{3}}+1+\frac{1}{1-\frac{341}{2,000}}\right\} \simeq 0.6748<1, \\
& 0.6748<1-\frac{1-b-\sqrt{1-2 b-b^{2}}}{2} \simeq 0.9821, \\
& 0.9872<1 .
\end{aligned}
$$

That is, conditions (1.12), (1.13), (1.14), (1.15), and (1.17) are not satisfied.

Figure 4, illustrates three different solutions of (2.15), as predicted, appeared to be oscillatory.

Notation It is worth noting that the improvement of condition (2.14) to the corresponding condition (1.12) is significant, approximately $93 \%$, if we compare the values on the leftside of these conditions. Also, the improvement compared to condition (1.14) (or (1.15)) is very satisfactory, around 46.3\%. Also, observe that conditions (1.14) and (1.15) do not lead to oscillation for first iteration. On the contrary, condition (2.14) is satisfied from the first iteration. This means that our condition is better and much faster than (1.14) and (1.15).

\subsection{Deviating difference inequalities}

A slight modification in the proofs of Theorems 1 and 2 leads to the following results about deviating difference inequalities.

Theorem 3 Assume that all conditions of Theorem 1 hold. Then

(i) the retarded difference inequality

$$
\Delta x(n)+p(n) x(\tau(n)) \leq 0, \quad n \in \mathbb{N}_{0}
$$




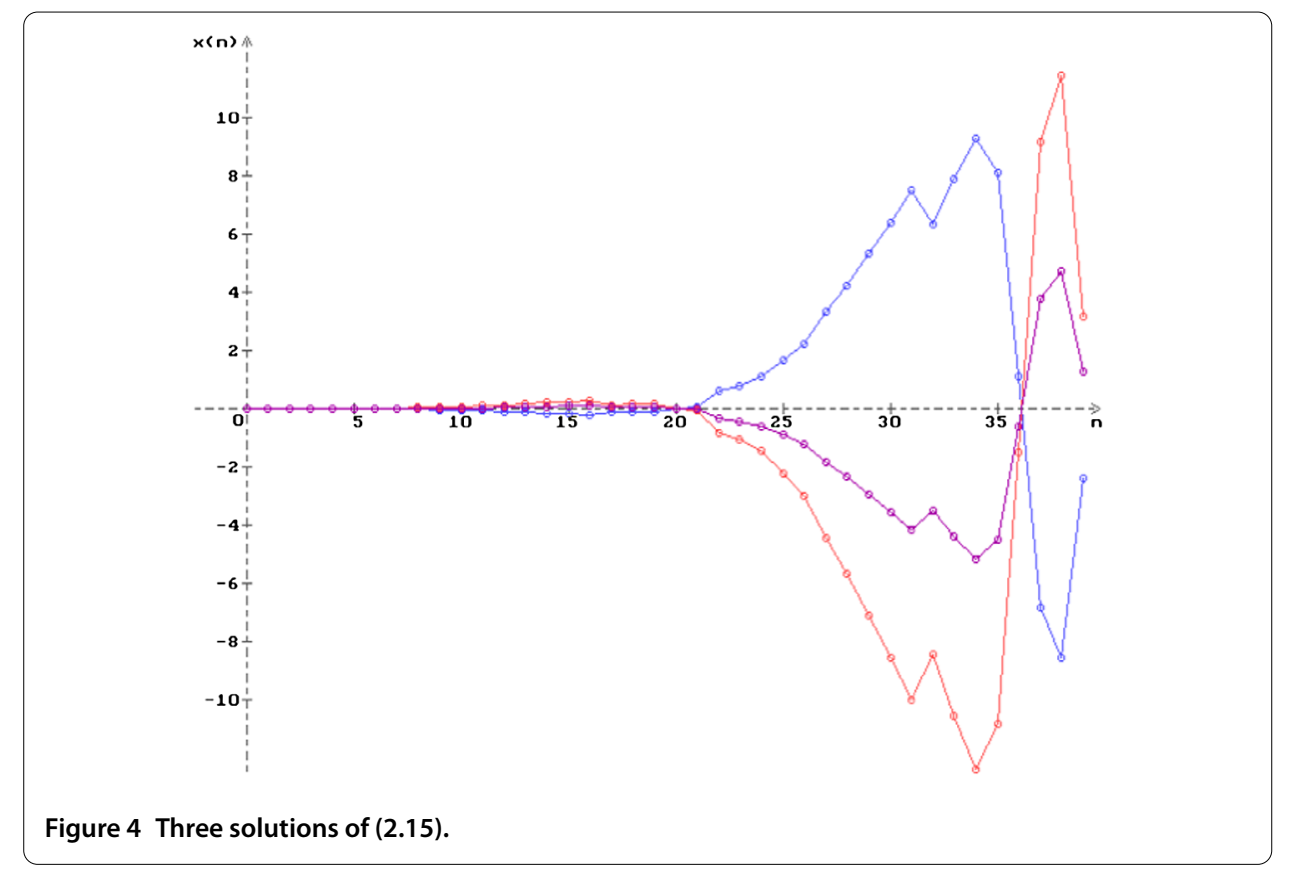

(ii) the retarded difference inequality

$$
\Delta x(n)+p(n) x(\tau(n)) \geq 0, \quad n \in \mathbb{N}_{0}
$$

has no eventually negative solutions.

Theorem 4 Assume that all conditions of Theorem 2 hold. Then

(i) the advanced difference inequality

$$
\nabla x(n)-q(n) x(\sigma(n)) \geq 0, \quad n \in \mathbb{N},
$$

has no eventually positive solutions;

(ii) the advanced difference inequality

$$
\nabla x(n)-q(n) x(\sigma(n)) \leq 0, \quad n \in \mathbb{N},
$$

has no eventually negative solutions.

\section{Competing interests}

The authors declare that they have no competing interests.

Authors' contributions

The authors declare that they have made equal contributions to the paper.

\section{Author details}

${ }^{1}$ Department of Electrical and Electronic Engineering Educators, School of Pedagogical and Technological Education (ASPETE), N. Heraklio, Athens, 14121, Greece. ${ }^{2}$ School of Electrical Engineering, Tel-Aviv University, Tel-Aviv, 69978, Israel.

\section{Acknowledgements}

The first author was supported by the Special Account for Research of ASPETE through the funding program

'Strengthening research of ASPETE faculty members'.

Received: 20 September 2016 Accepted: 15 February 2017 Published online: 27 February 2017 


\section{References}

1. Asteris, PG, Chatzarakis, GE: New oscillation tests for difference equations with non-monotone arguments. (to appear)

2. Braverman, E, Chatzarakis, GE, Stavroulakis, IP: Iterative oscillation tests for difference equations with several non-monotone arguments. J. Difference Equ. Appl. 21(9), 854-874 (2015)

3. Braverman, E, Karpuz, B: On oscillation of differential and difference equations with non-monotone delays. Appl. Math. Comput. 218, 3880-3887 (2011)

4. Chatzarakis, GE, Koplatadze, R, Stavroulakis, IP: Oscillation criteria of first order linear difference equations with delay argument. Nonlinear Anal. 68, 994-1005 (2008)

5. Chatzarakis, GE, Koplatadze, R, Stavroulakis, IP: Optimal oscillation criteria for first order difference equations with delay argument. Pacific J. Math. 235, 15-33 (2008)

6. Chatzarakis, GE, Philos, ChG, Stavroulakis, IP: Oscillations of first order linear difference equations with general delay argument. Portugal. Math. 66, 513-533 (2009)

7. Chatzarakis, GE, Stavroulakis, IP: Oscillations of difference equations with general advanced argument. Cent. Eur. J. Math. 10, 807-823 (2012)

8. Chen, M-P, Yu, JS: Oscillations of delay difference equations with variable coefficients. In: Proceedings of the First International Conference on Difference Equations, pp. 105-114. Gordon and Breach, London (1994)

9. Erbe, LH, Zhang, BG: Oscillation of discrete analogues of delay equations. Differential Integral Equations 2, 300-309 (1989)

10. Györi, I, Ladas, G: Linearized oscillations for equations with piecewise constant arguments. Differential Integral Equations 2, 123-131 (1989)

11. Koplatadze, RG, Kvinikadze, G: On the oscillation of solutions of first order delay differential inequalities and equations. Georgian Math. J. 3, 675-685 (1994)

12. Ladas, G, Philos, ChG, Sficas, YG: Sharp conditions for the oscillation of delay difference equations. J. Appl. Math. Simul. 2, 101-111 (1989)

13. Ladas, G: Explicit conditions for the oscillation of difference equations. J. Math. Anal. Appl. 153, $276-287$ (1990)

14. Li, X, Zhu, D: Oscillation of advanced difference equations with variable coefficients. Ann. Differ. Equ. 18, 254-263 (2002)

15. Stavroulakis, IP: Oscillation criteria for delay and difference equations with non-monotone arguments. Appl. Math Comput. 226, 661-672 (2014)

16. Tang, $\mathrm{XH}, \mathrm{Yu}, \mathrm{JS}$ : Oscillation of delay difference equations. Comput. Math. Appl. 37, 11-20 (1999)

17. Tang, XH, Zhang, RY: New oscillation criteria for delay difference equations. Comput. Math. Appl. 42, 1319-1330 (2001)

18. Yan, W, Meng, Q, Yan, J: Oscillation criteria for difference equation of variable delays. DCDIS Proceedings 3, 641-647 (2005)

19. Zhang, BG, Tian, CJ: Nonexistence and existence of positive solutions for difference equations with unbounded delay. Comput. Math. Appl. 36, 1-8 (1998)

\section{Submit your manuscript to a SpringerOpen ${ }^{\circ}$ journal and benefit from:}

- Convenient online submission

Rigorous peer review

Immediate publication on acceptance

- Open access: articles freely available online

- High visibility within the field

- Retaining the copyright to your article 